\title{
CLIMESCO: evolution of cropping systems as affected by climate change
}

\author{
Domenico Ventrella \\ Consiglio per la ricerca e la sperimentazione in agricoltura, Unità di ricerca per i sistemi colturali \\ degli ambienti caldo-aridi (CRA-SCA), Bari, Italy
}

\section{Context}

In this number of Italian Journal of Agronomy, seventeen scientific papers are published on the main results of the project CLIMESCO. This project was supported by three Italian Ministries (Ministero dell'Istruzione, dell'Università e della Ricerca, Ministero delle Politiche Agricole Alimentari e Forestali and Ministero dell'Ambiente e della tutela del territorio e del Mare) in the framework of Fondo Integrativo Speciale Ricerca (FISR, Special Integrated Research Fund). Most recent studies based on observed data and simulations of future climate conditions showed that the global increase of temperatures is most likely due to the increased concentration of Green House Gases. The effect of warming is unequally distributed around the globe, with some areas more sensitive to climate change than others, as the Mediterranean region. Climate change over this region is shown to be characterized by increasing temperatures and by relatively large changes in the frequency of extreme climatic events for both temperature and rainfall. The agricultural and food systems represent one of the most sensitive and vulnerable sectors of the area.

In general the increasing temperatures shorten the growing season for most field crops, with shorter time for biomass accumulation, thereby resulting in a lower yield. However, changes in yields were also dependent on crop distribution (e.g. summer and winter crops), crop type (e.g. C3 and C4 plants) and environmental conditions, as water and nutrient availability. To avoid or at least reduce negative effects of climate change several agronomic adaptation strategies for agriculture have been suggested. They include both short-term adjustments and long-term adaptations.

The short-term adaptation strategies are carried out at farm scale and include: optimization of sowing/transplanting time; selecting varieties and/or species among those available at present with more appropriate thermal time and vernalisation requirements and/or with

Correspondence: Domenico Ventrella, Consiglio per la ricerca e la sperimentazione in agricoltura, Unità di ricerca per i sistemi colturali degli ambienti caldo-aridi (CRA-SCA), via Celso Ulpiani 5, 70125 Bari, Italy.

E-mail: domenico.ventrella@entecra.it

Received for publication: 23 January 2011.

Accepted for publication: 30 January 2011.

CCopyright D. Ventrella, 2012

Licensee PAGEPress, Italy

Italian Journal of Agronomy 2012; 7:e.1

doi:10.4081/ija.2012.e1

This article is distributed under the terms of the Creative Commons Attribution Noncommercial License (by-nc 3.0) which permits any noncommercial use, distribution, and reproduction in any medium, provided the original author(s) and source are credited. increased resistance to heat waves and drought; optimization of amount and time of irrigation and/or other water management practices; optimization of fertiliser rates to maintain grain or fruit quality; applying the available technologies to conserve soil water (no-tillage, minimum tillage); using water resources of low quality (saline water).

The adaptation options feasible in midterm, where more or less expensive investments are required, can be summarized in: constitution, evaluation and introduction of new variety; structural enhancement of irrigation efficiency and changes in farming systems; adoption at farming/regional scale of new technologies to schedule the irrigation; adoption of advanced procedures based on remote sensing information; structural enhancement at regional/farming scale for using urban depurated water.

In a context characterized by climate change, high evaporative and transpirative demands, poor availability of water resources and/or decreasing of water quality for irrigation, the overall objective of CLIMESCO was to individuate integrated approaches for optimizing water resources use by maximizing the cropping systems WUE, approaches that could be utilized by political stakeholders in land planning activity.

\section{CLIMESCO project}

The research activity was carried out for two reference area in southern Italy: the Capitanata area in Apulia Region and the Diego Nivolelli basin in Sicily Region.

The following Italian research institutions were involved in CLIMESCO:

- Consiglio per la ricerca e la sperimentazione in agricoltura, Unità di ricerca per i sistemi colturali degli ambienti caldo-aridi (Coordinator).

- Università degli Studi di Bari, Dipartimento di Scienze AgroAmbientali e Territoriali.

- Consiglio Nazionale delle Ricerche, Istituto di Scienze dell'Atmosfera e del Clima, Sezione di Lecce.

- Università degli Studi di Palermo, Dipartimento dei Sistemi AgroAmbientali.

- Università degli Studi della Basilicata, Dipartimento di Scienze dei Sistemi Colturali, Forestali e dell'Ambiente.

- Università degli Studi di Foggia, Dipartimento di Scienze Agroambientali, Chimica e Difesa.

- Università degli Studi di Milano, Dipartimento di Produzione Vegetale

- Università di Bologna, Dipartimento di Scienze e Tecnologie Agroambientali.

- Università del Salento, Dipartimento di Scienze dei Materiali.

- Consiglio per la ricerca e la sperimentazione in agricoltura, Centro di ricerca per la cerealicoltura (Foggia).

- Consiglio Nazionale della Ricerca - Istituto di Scienze delle Produzioni Alimentari (Bari). 
The Project was structured in four workpackages (WP) with specific objectives, high degree of interaction and information exchange.

\section{WP1, identification of homogeneous areas}

The objective was to characterize the two areas starting from spatially distributed data concerning soil, climate and land use. Several techniques of data spazialization, clustering, geostatistical analysis, GIS was utilized to achieve homogeneous areas.

\section{WP2, climatic change}

The main objective was to provide temperature, precipitation and radiation scenarios according to several forecasted of green-housegases emission by using the General Circulation Models (GCM) and adopting dynamical and statistical downscaling procedures as regionalization techniques.

\section{WP3, optimization of water resources}

Field researches were carried out for agronomical studies regarding: irrigation management with saline water in Mediterranean environments at high desertification risk, cropping systems and water require- ments, agronomical strategies for soil leaching, temporal evolution of crop coefficients (Kc), crop resistance depending on climate change and evaluation of new procedures to determine hydrological soil parameters for soil water balance models and parameterization for simulation models about crop growth soil water balance.

\section{WP4, scenarios analysis}

This WP was the conclusive step of CLIMESCO. Simulations were performed at field and regional scale by using numerical models for simulating crops and cropping systems. Calibrated and tested crop and hydrological models were utilized in order to evaluate the effects that the future climatic scenarios will have on crop yields and to individuate the best agronomical strategy to optimize the use of water resources.

\section{Conclusions}

Several meeting and conferences about the vulnerability and adaptation capacity of the cropping systems to climate change were organized during the project. Extensive information and annual reports are available on the CLIMESCO home page (http://limesco.entecra.it). My special thanks go to all those people who through their work, both scientific and administrative, have made possible the achievement of the CLIMESCO objectives and in particular to the colleagues of CRA-SCA. 